\title{
The validation of a nutrition screening tool for children
}

\author{
H. McCarthy ${ }^{1,2}$, H. McNulty ${ }^{1}$, M. Dixon ${ }^{2}$ and M. J. Eaton-Evans ${ }^{1}$ \\ ${ }^{1}$ University of Ulster, Cromore Road, Coleraine BT52 1SA, UK and ${ }^{2}$ Department of Nutrition and Dietetic, \\ Manchester Children's Hospitals, Manchester M9 7AA, UK
}

Screening for nutrition risk using validated nutrition screening tools (NST) is well established as good practice in adult and elderly health care $^{(1)}$. Currently, there is no valid NST in the UK for use with children. Nursing staff working with children have a clear role in the identification of those at nutrition risk ${ }^{(2)}$; however, the lack of a NST limits the effectiveness with which nurses can undertake this role. The present study aimed to validate a new nurse-administered paediatric NST within an acute hospital.

Full ethical approval was obtained from the Salford and Trafford Research Ethics Committee before undertaking the current study. All children (aged 2-17 years) admitted to the study wards (two medical, two surgical) of a large paediatric centre in Manchester, UK, over a 4-month period in 2007-8 were screened using a newly-developed nurse-administered paediatric NST. The NST consisted of three scored elements: clinical diagnosis; nutritional intake: anthropometric measures; with an overall score of $\geq 4$ considered as 'at nutrition risk'. Of those screened a sample ( $n$ 238) also underwent full nutritional assessment by a registered dietitian. The full nutritional assessment consisted of a face-to-face interview to obtain dietary and social information and anthropometric measurements. Medical information was retrieved from case notes. Data were analysed using $\chi^{2}$ tests to compare groups and the $\kappa$ statistic to demonstrate agreement between the full nutritional assessment and NST.

Medical and surgical admissions were equally represented in the sample (51\% and $49 \%$ respectively), slightly more of the participants were male (57\%) and the mean age was 8.4 (SD 4.6) years. Nutrition risk was identified in $18 \%$ of the sample by both the NST (total score of $\geq 4$ ) and the full nutritional assessment. Compared with the full nutrition assessment the NST demonstrated $70 \%$ sensitivity and $91 \%$ specificity, with the $\kappa$ statistic value of 0.56 (95\% CI $0.40,0.71)$. The prevalence of nutrition risk identified was not significantly different between males and females (by either the NST or the full nutritional assessment), but was significantly higher (by both methods) in medical admission compared with surgical admission $\left(\chi^{2} 9.312, P=0.002\right.$ and $\chi^{2} 12.425, P<0.001$ respectively).

The results of this validation suggest that this new paediatric NST is valid and reliable for the identification of children requiring further nutritional assessment and appropriate intervention. It demonstrates moderate agreement with a full nutritional assessment.

1. Kondrup J, Allison SP, Elia M, et al. (2003) Clin Nutr 22, 415-421.

2. Royal College of Nursing (2006) Malnutrition: What Nurses Working with Children and Young People Need to Know and Do. London: RCN. 